\title{
Combined stellar evolution and spectroscopic modeling of massive stars
}

\author{
Jose H. Groh \\ Geneva Observatory, Geneva University, Versoix, Switzerland \\ email: jose.groh@unige.ch
}

\begin{abstract}
The morphological appearance of massive stars during their evolution and at the preSN stage is very uncertain, both from theoretical and observational perspectives. We recently developed coupled stellar evolution and atmospheric modeling of stars done with the Geneva and CMFGEN codes, for initial masses between 9 and $120 M_{\odot}$. We are able to predict the observables such as the high-resolution spectrum and broadband photometry. Here I discuss how the spectrum of a massive star changes across its evolution and before death. Our models allow, for the first time, direct comparison between predictions from stellar evolution models and observations of SN progenitors.
\end{abstract}

Keywords. stars: evolution, (stars:) supernovae: general, stars: mass loss

\section{The need for combined stellar evolution and atmospheric modeling}

Massive stars are essential constituents of stellar populations and galaxies in the near and far Universe. They are among the most important sources of ionizing photons, energy, and some chemical species, which are ejected into the interstellar medium through powerful stellar winds and during their extraordinary deaths as supernovae (SN) and long gamma-ray bursts (GRB). For these reasons, massive stars are often depicted as cosmic engines, because they are directly or indirectly related to most of the major areas of astrophysical research.

Despite their importance, our current understanding of massive stars is still limited. This inconvenient shortcoming can be explained by many reasons, such as uncertainties related to mass loss, rotation, binary interaction, and how to compare observations and models of massive stars. Here we focus on this last topic.

Our understanding of different classes of stars is often built by comparing evolutionary models and observations. However, mass loss may affect the spectra, magnitudes, and colors of massive stars, thus making the comparison between evolutionary models and observations a challenge. In addition to luminosity, effective temperature, and surface gravity, the observables of massive stars can be strongly influenced by a radiatively driven stellar wind that is characteristic of these stars. The effects of mass loss on the observables depend on the initial mass and metallicity, since they are in general more noticeable in MS stars with large initial masses, during the post-MS phase, and at high metallicities. When the wind density is significant, such as in Wolf-Rayet (WR) stars, the mass-loss rate, wind clumping, wind terminal velocity, and velocity law have a strong impact on the spectral morphology. This makes the analysis of massive stars a difficult task, and obtaining their fundamental parameters, such as luminosity and effective temperature, is subject to the uncertainties that comes from our limited understanding of mass loss and clumping. Furthermore, the definition of effective temperature of massive stars with dense winds is problematic and, while referring to an optical depth surface, it does not relate to a hydrostatic surface. This is caused by the atmosphere becoming extended, 
with the extension being larger the stronger the wind is. Stellar evolution models are able to predict the stellar parameters only up to the stellar hydrostatic surface, which is not directly reached by the observations of massive stars when a dense stellar wind is present. Since current evolutionary models do not thoroughly simulate the physical mechanisms happening at the atmosphere and wind, model predictions of the evolution of massive stars are difficult to be directly compared to observed quantities, such as a spectrum or a photometric measurement.

To improve the comparison between models and observations of massive stars, we recently devised coupled calculations of stellar evolution with the Geneva code and atmospheric and wind modeling with the CMFGEN code. This approach allows the investigation of stellar evolution based not only on interior properties, but also from a spectroscopic point of view. Essentially, the atmospheric models allow the physical quantities predicted by the stellar evolution model to be directly compared to observed features.

\section{Predicting the look of stars at the pre-SN stage}

Using the models described above, our group recently analyzed the properties of massive stars just before the SN explosion in a series of papers (Groh et al. 2013a, Groh et al. 2013b, Groh et al. 2013c, Groh et al. 2014, Groh 2014). Our models indicate that rotating stars with initial mass $\left(M_{\mathrm{ini}}\right)$ in the range $20-25 \quad M_{\odot}$ surprisingly end their lives as luminous blue variable (LBV) stars. The fate of single massive stars with $M_{\text {ini }}=9-120 M_{\odot}$ was investigated in Groh et al. 2013b, where we showed that massive stars, depending on their initial mass and rotation, can explode as red supergiants (RSG), yellow hypergiants (YHG), LBVs, and Wolf-Rayet (WR) stars of the WN and WO subtype. We applied these models to investigate the nature of the candidate progenitor of the $\mathrm{SN} \mathrm{Ib}$ iPTF13bvn, concluding that a single WR star with initial mass $\sim 31-35 M_{\odot}$ could explain the properties of the progenitor Groh et al. 2013c.

Figure 1 shows the different channels that link the spectral types of SN progenitors to the core-collapse SN types according to our models. For rotating models, we obtained the following types of SN progenitors: WO1-3 $\left(M_{\text {ini }} \geqslant 32 M_{\odot}\right)$, WN10-11 $\left(25<M_{\text {ini }}<\right.$ $\left.32 M_{\odot}\right), \operatorname{LBV}\left(20 \leqslant M_{\text {ini }} \leqslant 25 M_{\odot}\right)$, G1 Ia ${ }^{+}\left(18<M_{\text {ini }}<20 M_{\odot}\right)$, and RSGs $(9 \leqslant$ $\left.M_{\text {ini }} \leqslant 18 M_{\odot}\right)$. For non-rotating models, we found spectral types WO1-3 $\left(M_{\text {ini }}>\right.$ $\left.40 M_{\odot}\right)$, WN7-8 $\left(25<M_{\text {ini }} \leqslant 40 M_{\odot}\right)$, WN11h/LBV $\left(20<M_{\text {ini }} \leqslant 25 M_{\odot}\right)$, and RSGs $\left(9 \leqslant M_{\text {ini }} \leqslant 20 M_{\odot}\right)$. Our rotating models indicate that SN IIP progenitors are all RSG, SN IIL/b progenitors are $56 \%$ LBVs and $44 \%$ YHGs, SN Ib progenitors are $96 \%$ WN1011 and $4 \%$ WOs, and SN Ic progenitors are all WO stars. We find that not necessarily the most massive and luminous SN progenitors are the brighter ones in a given filter, since this depends on their luminosity, temperature, wind density, and how the spectral energy distribution compares to a filter bandpass. We find that SN IIP progenitors (RSGs) are bright in the $R I J H K_{S}$ filters and faint in the $U B$ filters. SN IIL/b progenitors (LBVs and YHGs), and SN Ib progenitors (WNs) are relatively bright in optical/infrared filters, while SN Ic progenitors (WOs) are faint in all optical filters.

We obtained a relationship between the absolute magnitude of RSGs in different filters and their initial mass (Groh et al. 2013b). This can be used to estimate the initial mass of progenitors detected in the pre-explosion images of SN IIP. Our method provides similar values of the initial mass of RSG as those found in the literature, given the uncertainties. We also investigated the detectability of SN Ib and Ic progenitors and argued that the WR stars that characterize the pre-SN phase are undetectable in the available pre-explosion images with the current magnitude limits. This is consistent with the current non-detection of WRs as progenitors of SN Ic in the available pre-explosion 


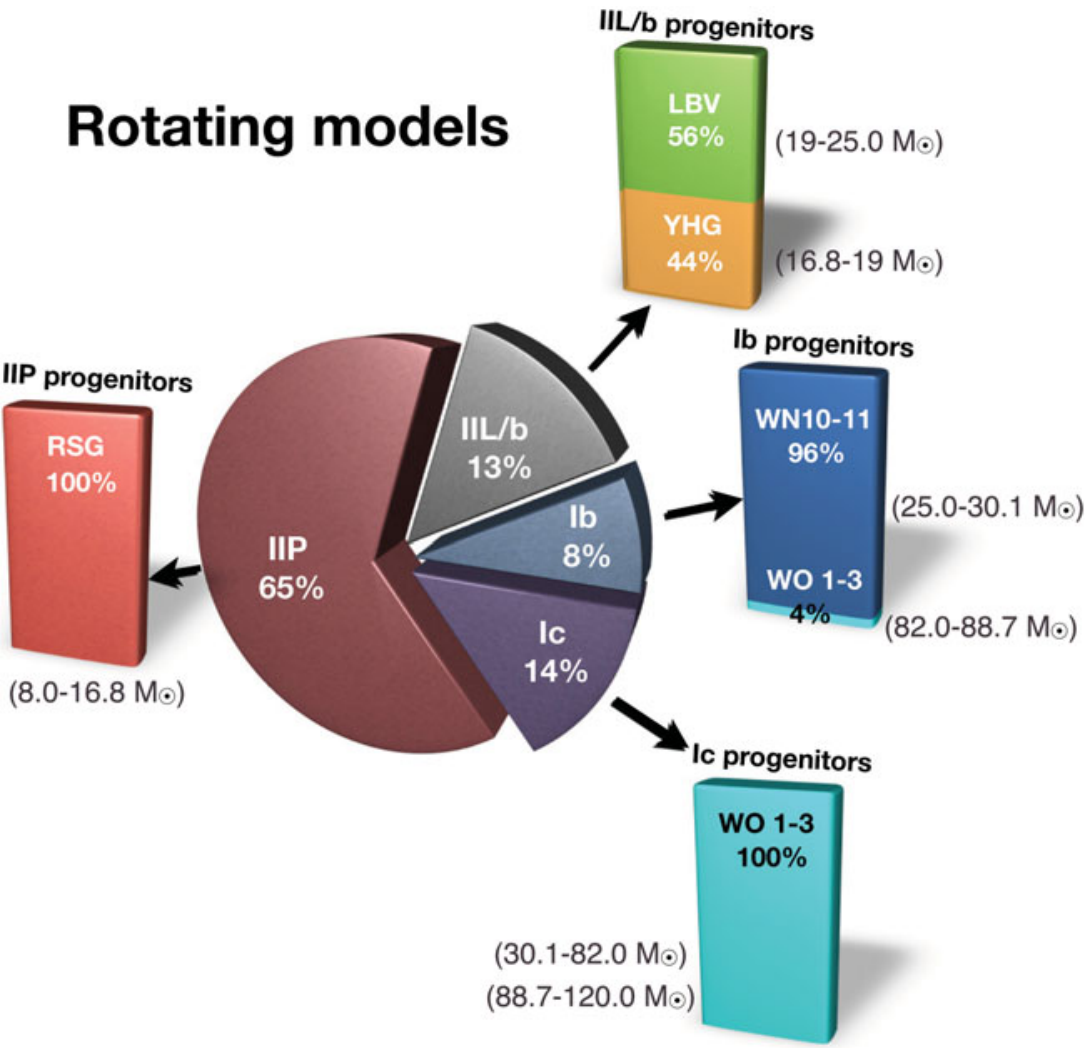

Figure 1. Diagram illustrating, for different core-collapse SN types, their relative rates and the types of progenitors and their respective frequencies. Initial mass ranges (indicated in parenthesis) and SN types are based on the criteria outlined in Georgy et al. (2012), assuming that the minimum amount of $\mathrm{He}$ in the ejecta to produce a $\mathrm{SN} \mathrm{Ib}$ is $0.6 M_{\odot}$.

images of Eldridge et al. (2013). Unlike the SN IIP progenitors, we find that the absolute magnitude in a given filter of SN Ic progenitors do not depend strongly on the initial mass. As a consequence, in the event that a type Ic progenitor is detected, our models indicate that it will be challenging to constrain its initial mass based on photometry (or spectroscopy) of the progenitor alone. To conclude, our analyses showed that it is crucial to produce an output spectrum out of evolutionary calculations to properly interpret the observations of massive stars at different evolutionary stages, in particular those with dense winds such as WR stars.

\section{References}

Eldridge, J. J. et al. 2013, ArXiv e-prints (1301.1975)

Georgy, C., Ekström, S., Meynet, G., et al. 2012, A\&A, 542, A29

Groh, J. H. 2014, A\& A, 572, L11

Groh, J. H., Georgy, C., \& Ekström, S. 2013a, A\&A, 558, L1

Groh, J. H., Hillier, D. J., Damineli, A., et al. 2009, ApJ, 698, 1698

Groh, J. H., Meynet, G., \& Ekström, S. 2013b, Aध\&A, 550, L7

Groh, J. H., Meynet, G., Ekström, S., \& Georgy, C. 2014, A\& A, 564, A30

Groh, J. H., Meynet, G., Georgy, C., \& Ekström, S. 2013c, A\&ऽA, 558, A131 